\title{
Induction of Regulatory B-Cells by Mesenchymal Stem Cells is Affected by SDF-1 $\alpha$-CXCR7
}

\author{
Yan Qin Zhihua Zhou Fang Zhang Yong Wang Bing Shen Yong Liu \\ Yifeng Guo Yu Fan Jianxin Qiu \\ Department of Urology, Shanghai First People's Hospital, School of Medicine, Shanghai Jiao Tong \\ University, Shanghai, China
}

\section{Key Words}

Mesenchymal stem cells (MSCs) • Regulatory B cells (Breg) • Immunomodulatory • CXCL12/ SDF-1 $\alpha$

\begin{abstract}
Background/Aims: Mesenchymal stem cells (MSCs) possess immunomodulatory properties on a diverse array of immune cell lineages, including regulatory $T$ and $B$ cells (Tregs and Bregs, respectively). However, their specific effects and mechanisms underlying induction of Bregs remain unclear. The immune regulatory function of MSCs is exerted through both cell-cell contact and the release of soluble factors. The main objective of this study was to examine the role of the SDF-1-CXCR4/CXCR7 axis in the secretory action of MSCs, and potential effects on the immunoregulatory function of these cells. Methods: MSCs were isolated from mouse bone marrow and characterized according to their multilineage differentiation potential and their surface antigen expression. CD19+ $B$ cells purified from mice splenocytes were co-cultured with MSCs at various ratios in the presence of LPS and $\alpha$ CD40. After 4 days, intracellular IL-10 production and cell surface $C D 1 d$ and $C D 5$ expression by $C D 19^{+} B$ cells were determined using flow cytometry, and the secretion of IL-10, IL-6, IgM, and IgG were assessed with ELISA. MSCs were treated with different concentrations of stromal derived factor-1 $\alpha$ (SDF- $1 \alpha$ ) stimuli or transiently overexpressed with CXCR7. and their cell viability and immune regulatory effects of MSCs on Bregs were assessed. Results: MSCs induced IL-10-producing regulatory B cells and primarily stimulated the $C D 1 d^{+} C D 5^{+} B$ cell subset of IL-10+Breg cells to express IL-10. IL-10, IL-6, and IgM secretion were additionally induced by MSCs. The CXCR7 pathway was required for MSC viability and the production of paracrine factors under SDF-1 $\alpha$ culture condition. Low concentrations of SDF- $1 \alpha$ promoted the immunomodulatory effect of MSCs, leading to a further increase in IL-10-producing regulatory B cells and IL-10 secretion. In contrast, high concentrations of SDF- $1 \alpha$ inhibited MSCs induction of IL-10+Breg cells. Notably, CXCR7 overexpression in MSCs reversed the inhibitory effect of high concentrations of SDF-1 $\alpha$ and promoted the immunomodulatory effect of these cells. Conclusion: MSCs induce IL-10+Breg cells, which contribute to the generation of an immunosuppressive environment. SDF-1 $\alpha$




\begin{tabular}{|c|c|c|}
\hline Cellular Physiology & Cell Physiol Biochem 2015;37:117-130 & \\
\hline and Biochemistry & $\begin{array}{l}\text { DoI: 10.1159/000430338 } \\
\text { Published online: August 17, } 2015\end{array}$ & $\begin{array}{l}\text { (0) } 2015 \text { S. Karger AG, Basel } \\
\text { www.karger.com/cpb }\end{array}$ \\
\hline
\end{tabular}

and its receptor, CXCR7 play important roles in the immunomodulatory function of MSCs by regulating their paracrine actions.

Copyright (c) 2015 S. Karger AG, Basel

\section{Introduction}

Mesenchymal stem cells (MSCs) have potential utility in various treatment strategies including immunoregulatory and regenerative therapies [1,2], and their effects of MSCs on immune cells is an important area of research. MSCs display broad immunomodulatory properties, both in vitro and in vivo [3-5]. The role of T-cells in graft rejection is fairly well documented, and the effects of MSCs on T-cells have been the focus of several studies to date. The finding that MSCs attenuate the T-cell response in graft rejection has led to their application as adjuncts to reduce transplant rejection [6]. However, the role of B-cells in transplant rejection was uncovered only recently, and the effect of MSCs on these cells has only just begun to be elucidated [7]. Inconsistent results have been reported, which are partly attributable to differences in experimental design between studies [8-11], highlighting the need for additional research in this area. Furthermore, the interactions of MSCs with the specific group of B-cells involved in regulating immune responses (regulatory B-cells or Bregs) are currently unknown.

Regulatory B cells negatively regulate immune responses via secretion of interleukin (IL)-10. The potent subsets of regulatory B cells have been functionally defined by their immune-suppressive actions in vitro and in vivo $[12,13]$. Mauri et al. described the splenic $\mathrm{CD} 21^{\text {hi }} \mathrm{CD} 23^{\text {hi }} \mathrm{CD} 1 \mathrm{~d}^{\text {hi }} \mathrm{T} 2$ marginal zone (MZ) precursor B cell group with confirmed regulatory functions [13]. IL-10-producing B cells, termed "B10" cells were characterized by the group of Tedder [14]. The latter are mainly detected within the $\mathrm{CD} 1 \mathrm{~d}^{\text {hi }} \mathrm{CD} 5^{+}$splenic B cell subset [14-16]. IL-10-producing Bregs are not present under normal physiological conditions, but generated during inflammatory processes [17]. Under these conditions, they play an important role in limiting inflammation and facilitating recovery as well as inducing tolerance [17]. In the current study, we initially investigated that the capacity of MSCs to generate Breg cells in the presence of LPS and $\alpha \mathrm{CD} 40$.

The immunoregulatory function of MSCs is exerted both through cell-cell contact and release of soluble factors, such as IL-10, IL-6, IL-2, IFN- $\gamma$, and TNF- $\alpha[4,16,18,19]$. The SDF1-CXCR4/CXCR7 axis is known to play an important role in the secretory action of MSCs. The chemokine stromal derived factor-1 $\alpha$ (CXCL12/SDF-1) is implicated in many biological processes, including stem cell biology, leucocyte trafficking, cancer, and inflammatory disorders [20-23]. CXCR4 is a typical chemokine receptor known to activate a variety of cellular processes, such as proliferation, apoptosis, survival, chemotaxis and differentiation $[24,25]$. Previous research has shown that human breast adipose-derived stem cells (HBASCs) transfected with CXCR4 can enhance the survival and quality of transplanted free fat tissues in nude mice [26]. CXCR7, an atypical chemokine receptor that binds CXCL12 and CXCL11 [27], has also been shown to associate with CXCR4 and regulate CXCR4/SDF1-mediated processes, such as the migration of plasmablasts during B-cell maturation [28]. However, the CXCR7-mediated cellular responses to CXCL12 are still largely unknown. Here, we investigated the effects of the SDF-1-CXCR7 axis on the cell viability, secretory activity, and immunoregulatory effects of MSCs in vitro. Our results support the potential utility of the crucial SDF-1-CXCR7 pathwayas an effective target for improving the beneficial effects of the immunomodulatory properties of MSCs.

\section{Materials and Methods}

MSCs culture and characterization

MSCs were cultured as described previously [29]. Briefly, bone marrow was harvested from the tibia of 8-10 week-old C57BL/6 mice, suspended in modified minimum essential Eagle's medium (DMEM, Gibco, 


\section{Cellular Physiology Cell Physiol Biochem 2015;37:117-130 \begin{tabular}{l|l|l} 
DOI: 10.1159/000430338 & (C) 2015 S. Karger AG, Basel
\end{tabular} \begin{tabular}{l|l} 
and Biochemistry Published online: August 17, 2015 & www.karger.com/cpb \\
\cline { 1 - 1 }
\end{tabular} \\ Qin et al.: MSCs Induce IL10+ Breg Cells}

Grand Island, NY, USA) containing 10\% fetal calf serum (FBS, Hyclone Laboratories Logan, UT, USA) and $1 \%$ penicillin-streptomycin (Gibco), and plated at a density of $1 \times 10^{6}$ cells $/ \mathrm{cm}$. MSCs were isolated using negative selection with a CD45+ microbead kit (MiltenyiBiotec, BergischGladbach, Germany). Isolated MSCs were characterized via flow cytometry using mesodermal and hematopoietic markers, and their adipogenic and osteogenic differentiation potential examined according to the procedure described by Carrion et al. [30].

\section{$B$-cell isolation and culture}

Splenic cells were acquired from C57BL/6 mice (6-8 weeks old) as described previously (Kuan, 2011). B lymphocytes were positively selected from total splenocytes with the magnetic-activated cell sorting (MACS) cell separation system (MiltenyiBiotec) using anti-mouse CD19 microbeads,according to the manufacturer's instructions. The selected cells were washed with phosphate buffered saline (PBS), suspended in RPMI 1640 (Gibco) supplemented with 10\% FBS (Gibco), cultured in 12-well flat-bottom plates (Costar, Cambridge, MA, USA) at a density of $2.5 \times 10^{6}$ cells $/ \mathrm{ml}$, and incubated at $37^{\circ} \mathrm{C}$ and $5 \% \mathrm{CO}_{2}$ in a Heracell incubator (Heraeus group, Hanau, Germany). The resulting B cell fraction contained >95\% CD19+ B cells as determined with flow cytometry (FCM).

\section{B-cell/MSCs co-culture}

MACS-purified CD19+ B cells activated in vitro using LPS $(10 \mu \mathrm{g} / \mathrm{ml})$ and an agonistic mAb against CD40 $(\alpha \mathrm{CD} 40,10 \mu \mathrm{g} / \mathrm{ml}$; clone FGK45) were co-cultured in 96-well plates at ratios of 1:1, 10:1, and 100:1 with MSCs suspended in RPMI 1640 (Gibco BRL, Life Technologies, Carlsbad, CA, USA) containing 10\% FBS (Gibco). After 4 days, CD19 ${ }^{+}$B cells were collected, and phenotypic analysis was performed using FCM. To determine whether the SDF-1-CXCR7 pathway plays a role in the immunomodulatory effect of MSCs on B cells, MSCs were stimulated with various concentrations of SDF-1 $\alpha$ (Millipore, Billerica, MA, USA) or overexpressed with CXCR7 for $24 \mathrm{~h}$, followed by co-cultured with activated B cells at a 1:1 ratio for 4 days. Phenotypic analyses of CD19+ B cells were performed using FCM.

\section{Flow cytometry}

FCM was performed as described previously [31]. Cells were washed twice and suspended in fluorescence activated cell sorter (FACS) buffer containing 2\% FBS and $0.1 \%$ sodium azide in PBS. Cells were subsequently incubated with the various antibodies according to the manufacturers' instructions. Antibodies used for labeling were FITC-labeled anti-Sca-1, anti-CD29 and anti-CD45 (BD Bioscience), PElabeled anti-CD44 and anti-CD11b (BD Bioscience, San Jose, CA, USA), APC-labeled anti-mouse anti-CD90 and anti-CD19 (BD Bioscience), FITC-labeled anti-mouse CD1d (BD Pharmingen, San Diego, CA, USA), PerCP-Cy5.5-labeled anti-mouse CD5 (eBioscience, San Diego, CA, USA), and FITC-labeled anti-CXCR4 and anti-CXCR7 (eBioscience). Flow cytometry was performed on a FACSC alibur (Becton Dickinson, San Jose, CA, USA) and analyzed with FlowJo software (Tree Star, Ashland, OR, USA).

Intracellular staining for IL-10 was performed as described in a previous report [10]. Activated B cells were co-cultured with various ratios of MSCs suspensions. After 4 days of incubation, cells were washed with PBS containing 1\% FBS and stained with APC-labeled anti-mouse CD $19 \mathrm{mAb}$ for $30 \mathrm{~min}$ at $4^{\circ} \mathrm{C}$ in the dark. Cells were re-washed in PBS with 1\% FBS and fixed in $4 \%$ paraformaldehyde for $20 \mathrm{~min}$ at $4{ }^{\circ} \mathrm{C}$ in the dark. Subsequently, cells were permeabilized with PBS buffer containing 1\% FBS and 0.1\% saponin and stained with PE-labeled anti-mouse IL-10 (eBioscience) at $4^{\circ} \mathrm{C}$ in the dark, followed by washing and analysis with FCM.

\section{Measurement of secreted cytokines and immunoglobulins}

MACS-purified CD19+ B cells were co-cultured with MSCs for 4 days, and cell-free culture supernatants assayed for murine IL- 6 and IL-10 using OptEIA ELISA kits (eBioscience) and mouse IgM and IgG using ELISA quantification kits (Bethyl Laboratories, Inc., Montgomery, USA) according to the manufacturers' instructions. Absorbance of assay plates was measured at $450 \mathrm{~nm}$ in a Bio-Rad (Hercules, CA, USA) 3550UV microplate reader. Production of IL-10, IL-6, IL-7, IL-4, IFN- $\gamma$, and TNF- $\alpha$ in the supernatant fractions of MSCs was determined with the Bio-Plex cytokine assay (Bio-Rad, Hercules, CA), in keeping with the manufacturer's instructions. 


\section{Cellular Physiology Cell Physiol Biochem 2015;37:117-130 \begin{tabular}{l|l|l} 
DOI: 10.1159/000430338 & (C) 2015 S. Karger AG, Basel
\end{tabular} \begin{tabular}{l|l|} 
and Biochemistry Published online: August 17, 2015 & www.karger.com/cpb \\
\cline { 1 - 2 }
\end{tabular} \\ Qin et al.: MSCs Induce IL10+ Breg Cells}

\section{Western blotting}

MSCs were washed with ice cold PBS and harvested in lysis buffer containing proteinase inhibitors. Proteins were separated onsodium dodecyl sulfate polyacrylamide gels (SDS-PAGE) and transferred onto polyvinylidene difluoride membranes. Membranes were blocked with TBS-T buffer containing 5\% nonfat dry milk and incubated overnight at $4^{\circ} \mathrm{C}$ with primary antibodies against CXCR4 (Santa Cruz Biotechnology, CA, USA), CXCR7 (R\&D Systems, Minneapolis, MN, USA), or $\beta$-actin (Santa Cruz Biotechnology), followed by washing and incubation with horseradish peroxidase (HRP)-conjugated secondary antibodies. Immune complexes were visualized by enhanced chemiluminescence (ECL).

\section{Transient cell transfection}

MSCs were transfected with an expression vector containing the mouse CXCR7 openreading frame, pUN01-mCXCR7, or an empty vector (pUN01) (InvivoGen, San Diego, CA, USA). $2 \times 10^{6}$ MSCs were plated in six-well plates and cultured in DMEM containing 10\% FBS until cell density reached $75 \%$ confluence. Plasmid DNA was diluted in opti-MEM medium (Gibco) and transfected into MSCs using Lipofectamine (Gibco). In each experiment, transfection efficiency was assessed using western blot. The functional characteristics of CXCR7-transfected MSCs were further examined using cell viability, ELISA assay and co-cultured assay.

Cell viability and cytotoxicity assays

MSCs $\left(2.5 \times 10^{5}\right)$ were seeded in 24-well plates and incubated with different concentrations of SDF- $1 \alpha$. The CXCR7 inhibitor, CCX771 $(1 \mu \mathrm{M}$, synthesized by ChemoCentryx, Mountain View, CA, USA), or the CXCR4specific antagonist, AMD3100 (5 $\mu$ M, Sigma-Aldrich), was added, and cell proliferation was measured after 0-4 days using the 3-(4,5-dimethyl-2-thiazolyl)-2,5-diphenyl-2H-tetrazolium bromide (MTT) assay. Optical density (OD) was measured at $490 \mathrm{~nm}$. Cell viability was evaluated after $48 \mathrm{~h}$ of culture with FCM using Annexin V-APC-Propidium Iodide (PI) staining, as described previously [32]. Cytotoxicity was additionally determined by measuring the amunt of dehydrogenase (LDH) released in cell culture medium using an LDH assay kit (Sigma) via recording absorption at $340 \mathrm{~nm}$ according to the protocolof the manufacturer.

\section{Transwell experiments}

Transwell chambers with $0.4 \mu \mathrm{m}$ pore membranes (Costar) were used to physically separate stimulated B cells from MSCs. Activated B cells $\left(1 \times 10^{6}\right)$ were seeded in the upper chamber and MSCs overexpressing CXCR7 in the lower chamber at a 1:1 ratio. After four days, phenotypic analysis of CD19+ $\mathrm{B}$ cells was performed using FCM.

Statistical analysis

Results are expressed as mean \pm SD. Experiments were repeated at least three times. The KruskalWallis or the Mann-Whitney test was performed for statistical comparisons using Graph-Pad Prism ${ }^{\mathrm{TM}}$ software (Graphpad, San Diego, CA, USA). $P$ values $<0.05$ were considered statistically significant.

\section{Results}

\section{Characteristics of mouse MSCs}

After the third passage, MSCs in culture exhibited a larger and clostridial phenotype (Fig. 1A). Under the appropriate stimuli, MSCs differentiated into adipocytes and osteoblasts (Fig. $1 \mathrm{~B}$ and $\mathrm{C}$, respectively), demonstrating multi-lineage differentiation potential. Cultivated MSCs strongly expressed the mesodermal surface markers CD90, CD29, SCA-1 and CD44, but not the hematopoietic markers, CD45 and CD11b (Fig. 1D).

MSCs induce IL-10+ B regulatory cells

To investigate the effect of MSCs on activated of murine B lymphocytes, MACS-purified CD19+ B cells stimulated for 72h with a combination of LPS and $\alpha$ CD40 were co-cultured with MSCs at different ratios. CD19+ B cells in the presence of stimuli incubated with MSCs at ratios of 1:1 and 1:10 displayed an increased proportion of IL-10 ${ }^{+}$Breg cells, compared with CD19 ${ }^{+}$B cells cultured without MSCs (Fig. 2A and B). Limited production of cytokines (IL-6 
A

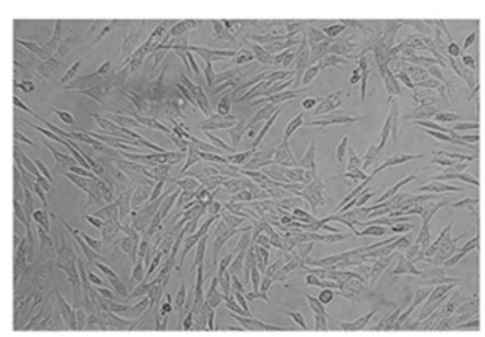

$\mathrm{B}$

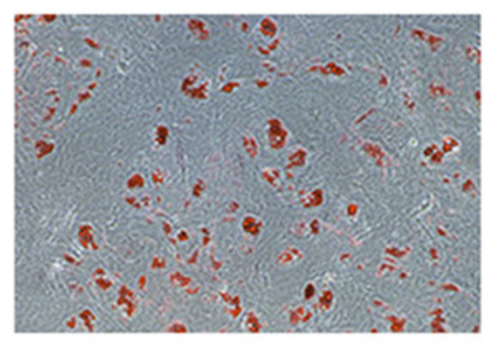

C

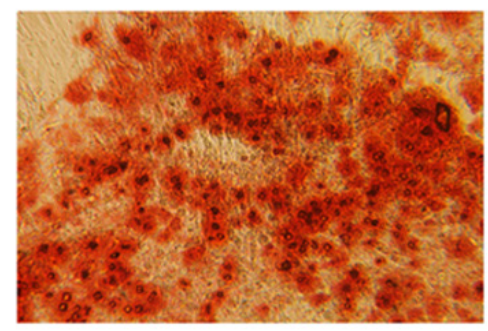

D
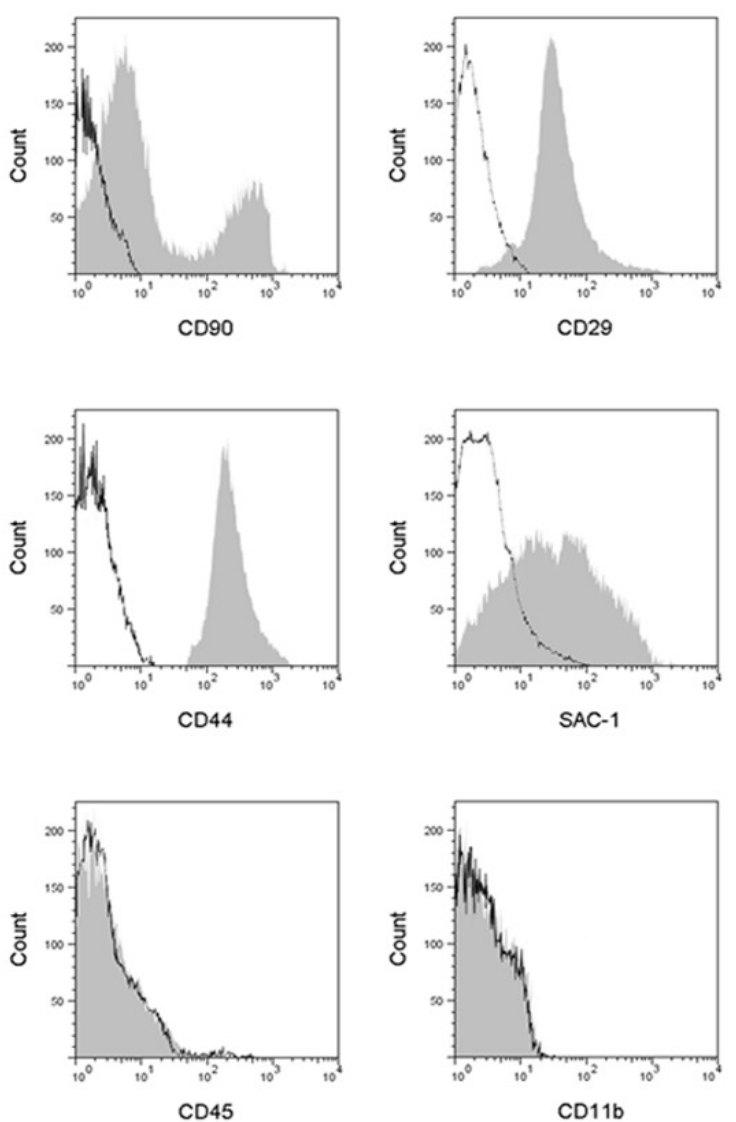

Fig. 1. Characteristics and differentiation potential of MSCs isolated from mouse bone marrow. (A) After three passages, MSC monolayer cultures were larger and clostridial in form in (100×). (B) Oil Red O staining showed accumulation of oil droplets in cells, indicating MSC differentiation into adipocytes.(C) Alizarin Red $\mathrm{S}$ staining disclosed osteocyte lineage differentiation potential of MSCs. (D) Phenotypic characterization of murine MSCs at passage 3 to 4 via FACS analysis. A representative histogram for each antigen is shown.

and IL-10) was observed in the cultures of MSC alone, while co-culture of MSCs with activated $B$ cells at the lower ratios (1:1 and 1:10 but not 1:100) resulted in increased secretion of IL-6 and IL-10 into culture supernatants (Fig. 2C and D).

We additionally examined the immunoglobulin (Ig) production ability of CD19+ B cells incubated without and with MSCs at ratios of 1:1, 10:1, and100:1 in the presence of LPS and $\alpha C D 40$. Notably, IgM secretion was increased in the supernatant fractions of co-cultures of B cells and MSCs at the lower ratios (1:1 and 10:1, but not 100:1), whereas IgG production remained unaffected (Fig. 2E and F).

The MSCs primarily stimulated the CD1 $\mathrm{d}^{+}$marginal zone (MZ) subset of IL- $10^{+}$Breg cells ( $>99 \%$ of total IL- $10^{+}$cells) to express IL-10, and $20-30 \%$ of the CD1 $\mathrm{d}^{+}$cells were CD5 ${ }^{+}$ B10-like cells (Fig. 2G and H).

\section{SDF- $1 \alpha$ regulates $M S C$ viability through CXCR7}

The SDF-1-CXCR4/CXCR7 axis plays an important role in chemotaxis, viability, and paracrine actions of MSCs. To determine the effects of SDF-1 on cell viability, MSCs were cultured with different concentrations of SDF- $1 \alpha$. Low concentrations of SDF- $1 \alpha(0.1 \mu \mathrm{g} / \mathrm{ml})$ stimulated cell proliferation and had no effect on cell viability (Fig. 3A and B). In contrast, higher concentrations of SDF- $1 \alpha(\geq 0.5 \mu \mathrm{g} / \mathrm{ml})$ significantly inhibited MSC proliferation and

\section{KARGER}


A

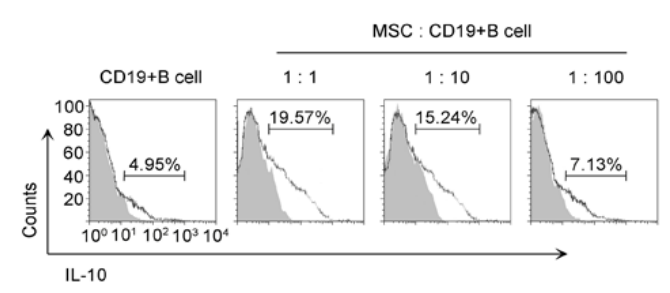

B

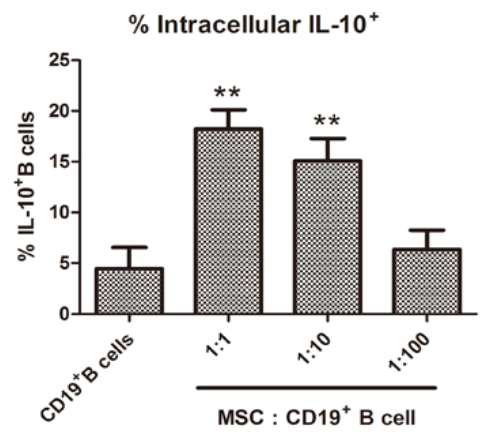

G

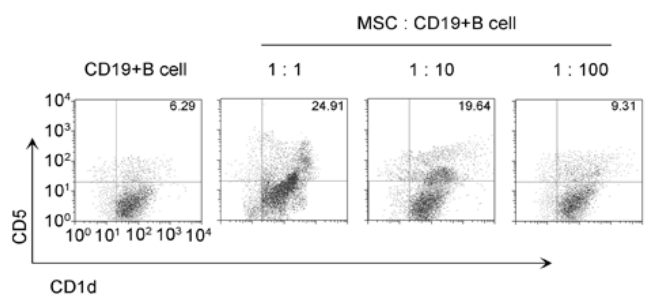

$\mathrm{H}$

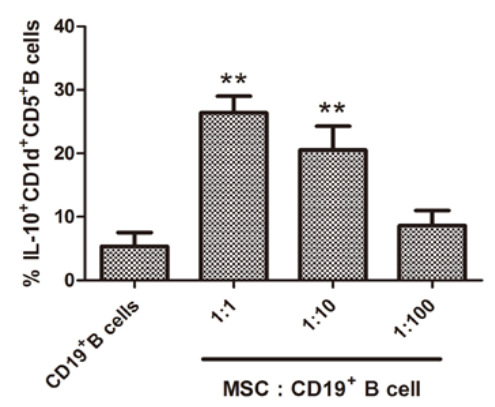

C

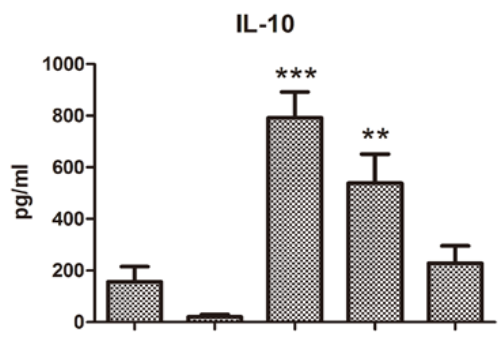

D

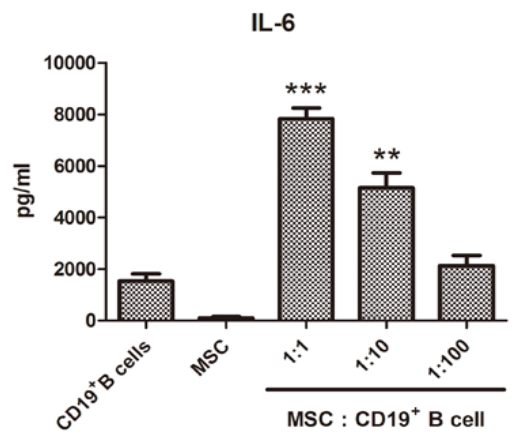

E

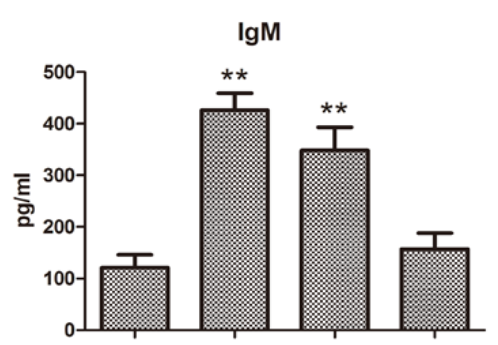

F

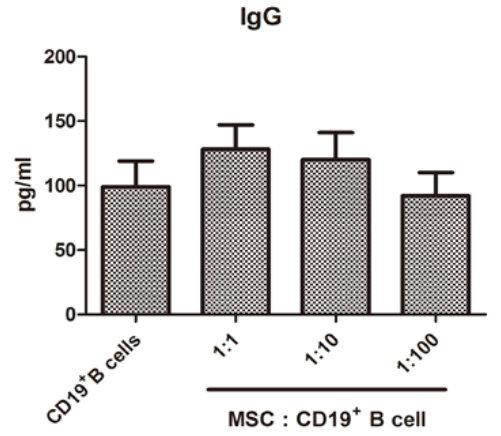

Fig. 2. MSCs stimulate Breg cell proliferation and cytokine secretion in supernatants of CD19+/MSCs co-culture. MACS-purified CD19+ B cells and MSCs were cultured for 4 days at the indicated ratios with B cells alone as controls. Cells were isolated and stained with CD19 mAb, or permeabilized and incubated with PE-conjugated anti-mouse IL-10 for intracellular staining. PerCP-Cy5.5-conjugated anti-mouse CD5 and FITC-conjugated anti-mouse CD1d were used for surface staining. Representative flow cytometric plots (A and G) are shown. Histograms showing the percentage of IL-10-producing (B) and IL- $10^{+} \mathrm{CD} 5^{+} \mathrm{CD} 1 \mathrm{~d}^{+}$cells $(\mathrm{H})$ among $\mathrm{CD} 19^{+} \mathrm{B}$ cells. Supernatants from co-cultures of CD19+ B cells and MSCs co-culture were collected at the end of the culture period and analyzed for the various secreted factors using ELISA. The levels of IL-10 (C), IL-6 (D), IgM (E), and IgG (F) are shown. Data shown were derived from four independent experiments (mean \pm SD). Significant differences, compared with $C D 19^{+}$B cells alone are shown ${ }^{* *} \mathrm{p}<0.01{ }^{* *} \mathrm{p}<0.001$ (in all panels). 


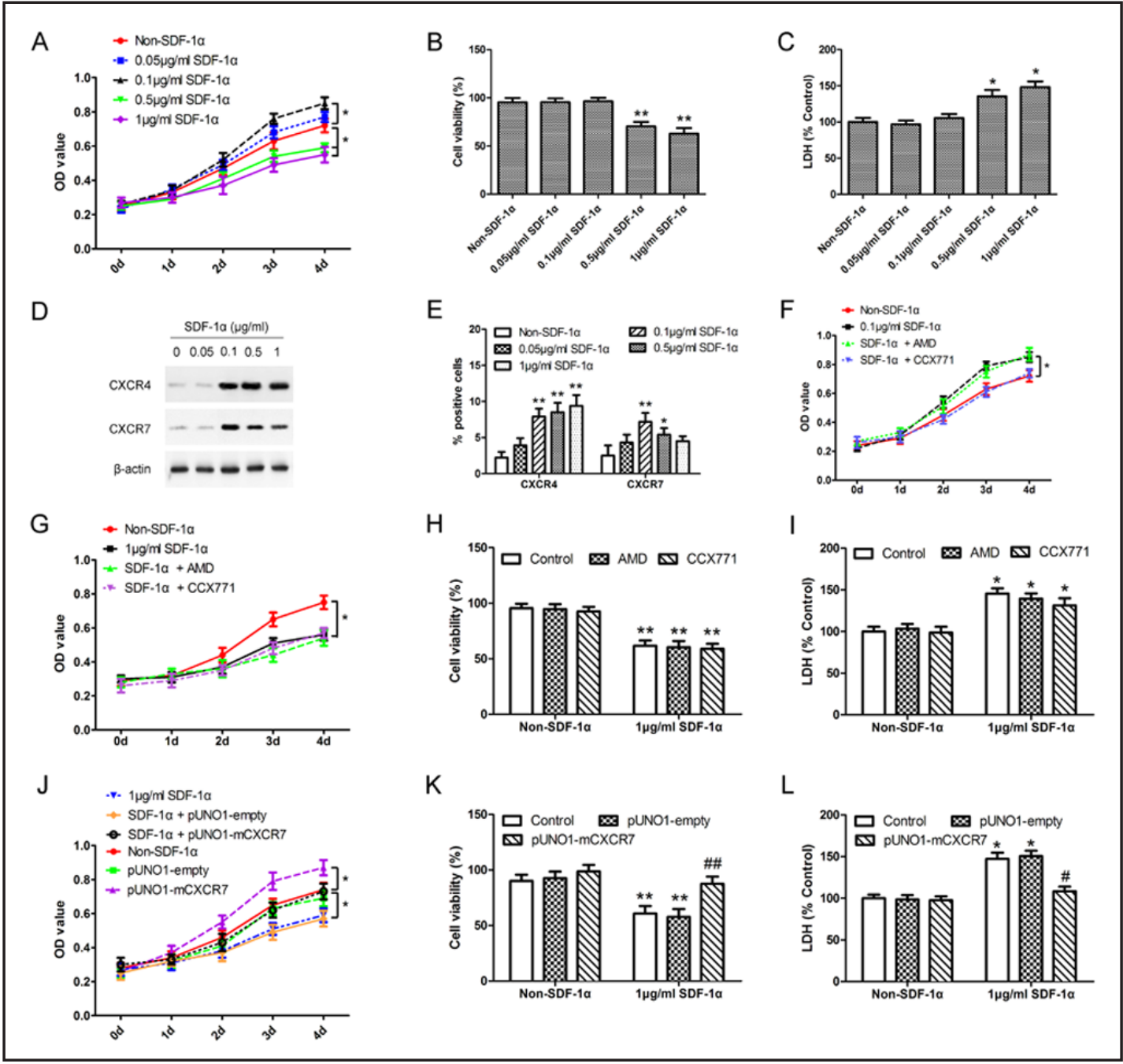

Fig. 3. Role of the CXCR4/CXCR7 receptors on SDF-1 $\alpha$-regulated MSC viability. (A) Proliferation of MSCs exposed to chemokine SDF- $1 \alpha(0.05,0.1,0.5,1 \mu \mathrm{g} / \mathrm{ml})$ after $0,1,2,3$ and 4 days, measured with the MTT-assay. Non-SDF-1 $\alpha$ treated cells served as the control. Mean optical density (OD) values at $490 \mathrm{~nm}$ from four replicates are depicted in the graph. (B) Cell viability was measured via Annexin V-PI staining and LDH assay used to assess membrane damage (C) in MSCs incubated with or without SDF- $1 \alpha(0.05,0.1,0.5,1 \mu \mathrm{g} / \mathrm{ml})$ for $48 \mathrm{~h}$. Results are presented as the mean \pm SD of four independent experiments. (D) MSCs at passage 3 were exposed to SDF- $1 \alpha(0.05,0.1,0.5,1 \mu \mathrm{g} / \mathrm{ml})$. CXCR4 and CXCR7 protein expression was detected using Western blot analysis, with $\beta$-actin as a control. (E) Extracellular expression of CXCR4 or CXCR7 in MSCs exposed to SDF- $1 \alpha(0.05,0.1,0.5,1 \mu \mathrm{g} / \mathrm{ml})$ detected using flow cytometry. $* \mathrm{p}<0.05,{ }^{* *} \mathrm{p}<0.01$, vs non- treated cells. MSCs were treated with SDF-1 $\alpha(0.1 \mu \mathrm{g} / \mathrm{ml}, \mathrm{F}$; or $1 \mu \mathrm{g} / \mathrm{ml}, \mathrm{G})$ together with the CXCR7 inhibitor, CCX771, or the CXCR4-specific antagonist, AMD3100, respectively. Non-SDF-1 $\alpha$ treated cells served as the control. MSCs Proliferation was measured with the MTT assay. *, p<0.05, vs non-SDF- $1 \alpha$ treated cells; (H and I) MSCs were treated with or without SDF-1 $\alpha(1 \mu \mathrm{g} / \mathrm{ml})$ and the CXCR7 inhibitor, CCX771, or the CXCR4-specific antagonist, AMD3100. Cell viability (H) and LDH release (I) were measured. (J, K and L) MSCs were transiently overexpressed with CXCR7 using pUN01-mCXCR7 or pUN01-empty vector, and incubated with or without SDF- $1 \alpha(1 \mu \mathrm{g} / \mathrm{ml})$. Proliferation of MSCs with the MTT assay (J) and cell viability (K) and LDH release (L) was measured. ${ }^{*} \mathrm{p}<0.05,{ }^{* *} \mathrm{p}<0.01$ vs Control; \#\# $\mathrm{p}<0.01$ vs $1 \mu \mathrm{g} / \mathrm{ml}$ SDF- $1 \alpha$ treated cells.

suppressed cell viability. LDH release of MSCs under SDF-1 $\alpha$ culture conditios was further evaluated. Increased cytotoxicity was observed upon treatment with high concentrations of SDF- $1 \alpha(\geq 0.5 \mu \mathrm{g} / \mathrm{ml})$ (Fig. 3C). 


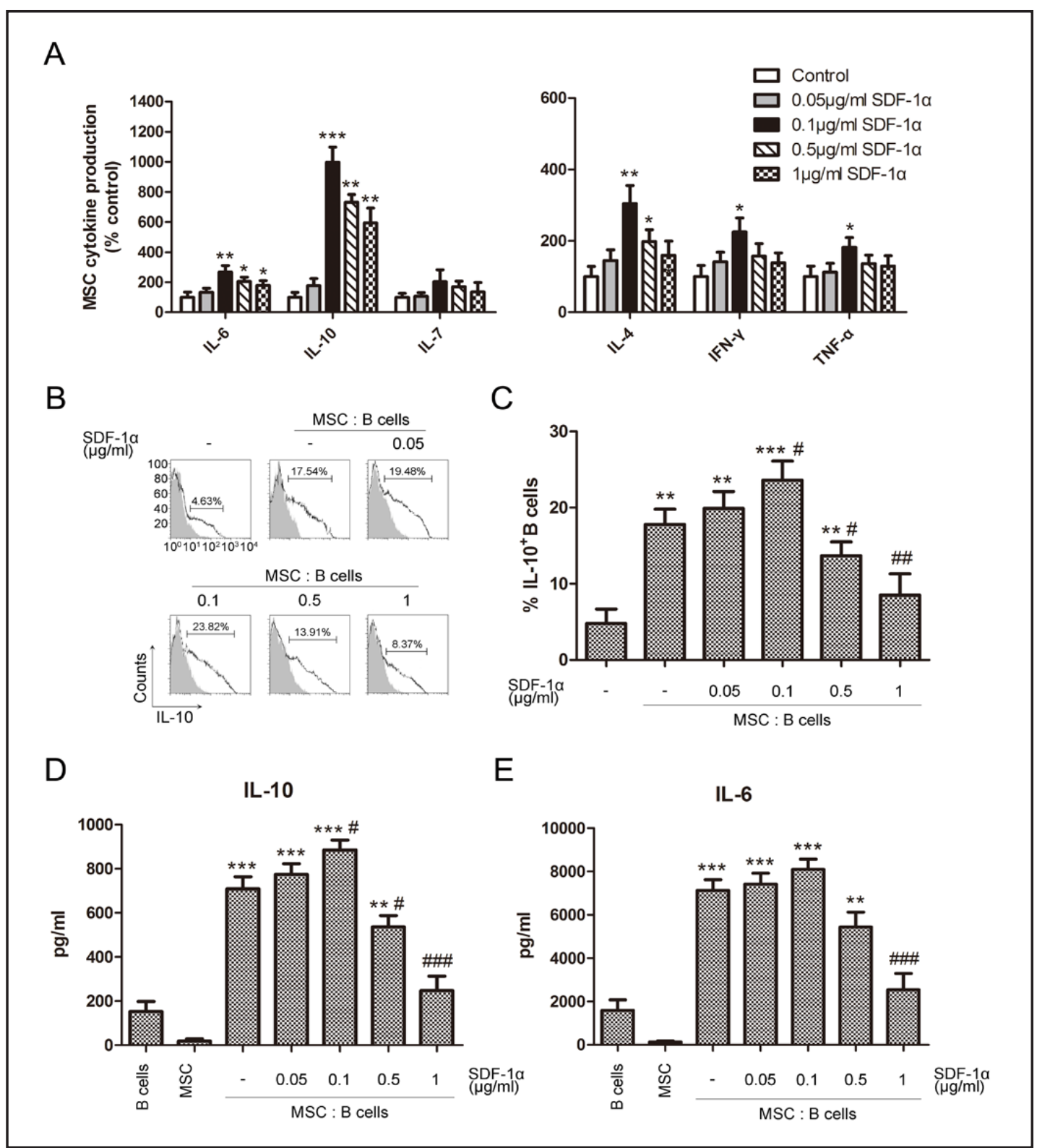

Fig. 4. Effects of SDF- $1 \alpha$ on MSC-induced IL-10+Breg cells. (A) MSC cytokine production after stimulation with or without SDF- $1 \alpha(0.05,0.1,0.5,1 \mu \mathrm{g} / \mathrm{ml})$. MSCs were stimulated with SDF- $1 \alpha$, after $24 \mathrm{~h}$, co-cultured with B cells at a 1:1 ratio. Cultured cells were isolated, stained with CD19 mAb, permeabilized, stained using IL-10 mAb, and analyzed with flow cytometry. Representative flow cytometric plots (B) and percentage of IL-10+ cells (C) are shown. Levels of IL-10 (D) and IL-6 (E) were detected directly in culture supernatant using ELISA. Data derived from three independent experiments (mean \pm SD) are shown. *, \# p<0.05, **, \#\# p<0.01, ***, \#\#\# p<0.001, * vs the control group, \# vs the B cell : MSC group.

To determine whether the effects of SDF-1 are mediated via CXCR7 and CXCR4, we initially determined the expression patterns of these receptors in MSCs treated with SDF- $1 \alpha$. Western blot analysis showed that at concentrations $\geq 0.1 \mu \mathrm{g} / \mathrm{ml}$, SDF- $1 \alpha$ upregulated both CXCR4 and CXCR7 (Fig. 3D). However, at SDF- $1 \alpha$ concentrations $\geq 0.5 \mu \mathrm{g} / \mathrm{ml}$, CXCR7 levels started to decrease gradually. FCM analysis further disclosed that the number of CXCR4- or CXCR7-positive cells was significantly higher in MSCs exposed to $\geq 0.1 \mu \mathrm{g} / \mathrm{ml}$ SDF- $1 \alpha$ than non-treated MSCs. At SDF- $1 \alpha$ concentrations $\geq 0.5 \mu \mathrm{g} / \mathrm{ml}$, the number of CXCR7-positive cells started to decrease gradually (Fig. 3E). 
A
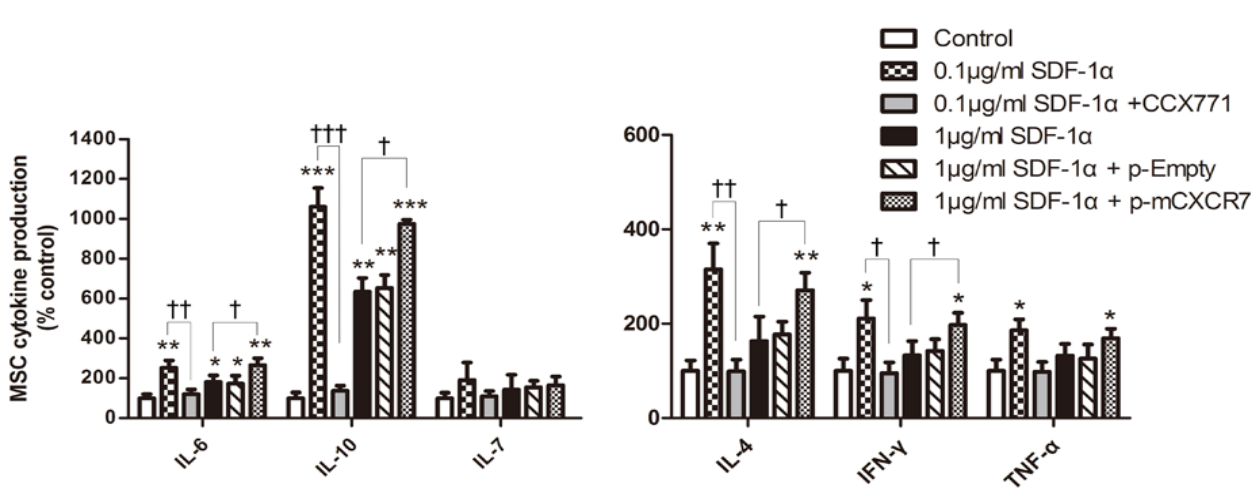

B

C
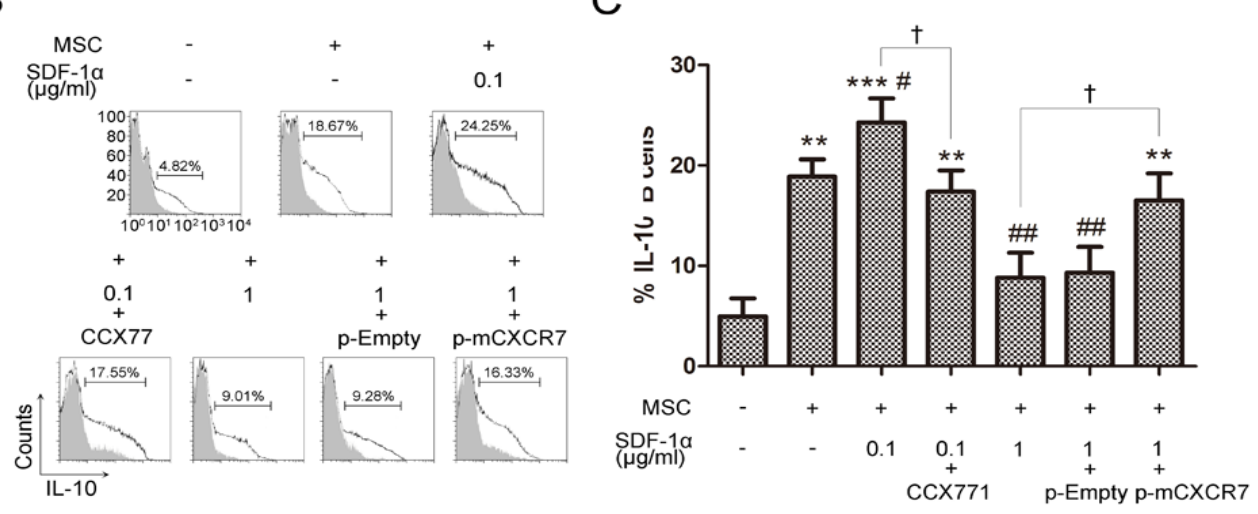

D

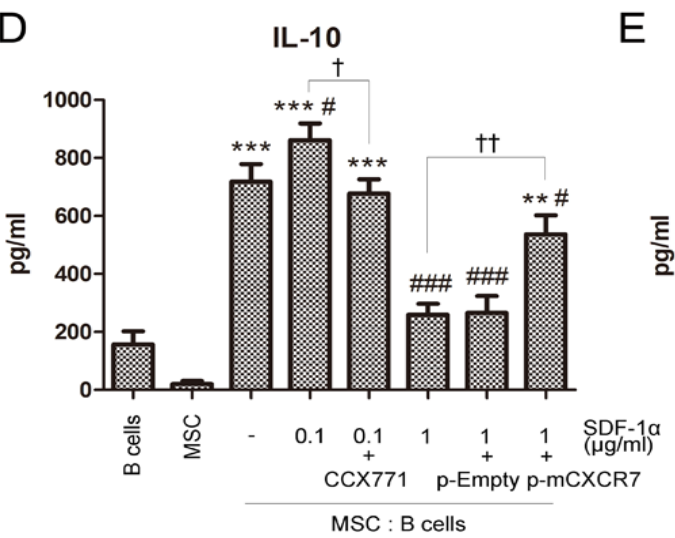

$E$

IL-6

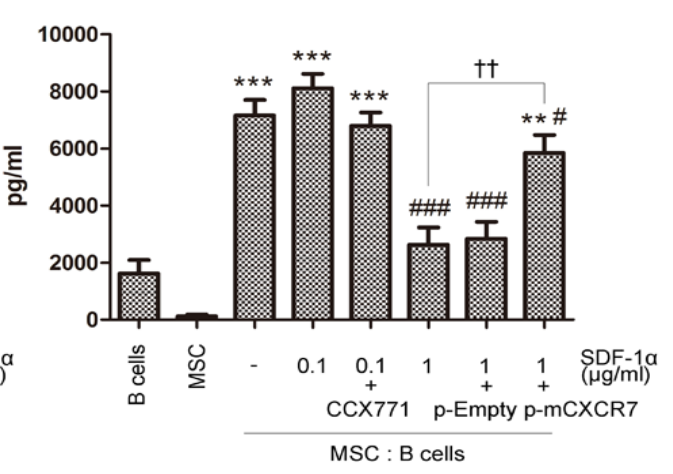

Fig. 5. Effects of SDF-1-CXCR7 on MSC-induced IL-10+Breg cells. (A) MSC cytokine production after stimulation with SDF- $1 \alpha(0.1 \mu \mathrm{g} / \mathrm{ml})$ and the CXCR7 inhibitor CCX771, or SDF- $1 \alpha(1 \mu \mathrm{g} / \mathrm{ml})$ and pUN01-mCXCR7. MSCs were stimulated with various stimuli, and after $24 \mathrm{~h}$, co-cultured with B cells at a 1:1 ratio. The cultured cells were isolated, stained with CD19 mAb, permeabilized, and stained using IL-10 mAb, followed by flow cytometry analysis. Representative flow cytometric plots (B) and the percentage of IL-10+ cells (C) are shown. Levels of IL-10 (D) and IL-6 (E) were detected directly in culture supernatants using ELISA. Data derived from three independent experiments (mean $\pm \mathrm{SD}$ ) are shown.*,, , \# p<0.05, **, ††, \#\# p<0.01, ***, †††, \#\#\# p<0.001, * vs the control group, \# vs the B cell : MSC group.

Treatment of MSCs with the CXCR7 inhibitor, CCX771, but not the CXCR4-specific antagonist, AMD3100, completely suppressed the stimulatory effect of low $(0.1 \mu \mathrm{g} / \mathrm{ml})$ concentrations of SDF- $1 \alpha$ on MSC proliferation (Fig. 3F). However, at high SDF- $1 \alpha(1 \mu \mathrm{g} / \mathrm{ml})$, neither inhibitor had an effect on MSC proliferation (Fig. 3G). Furthermore, neither CCX771 
A

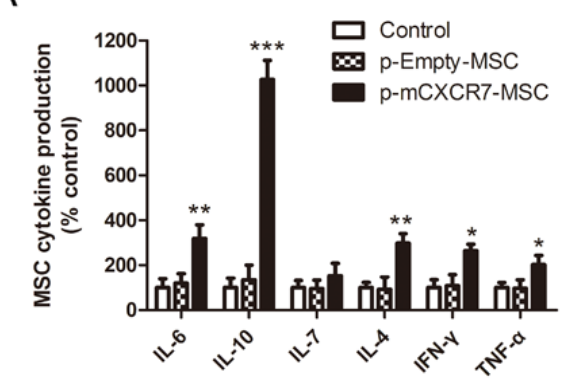

B
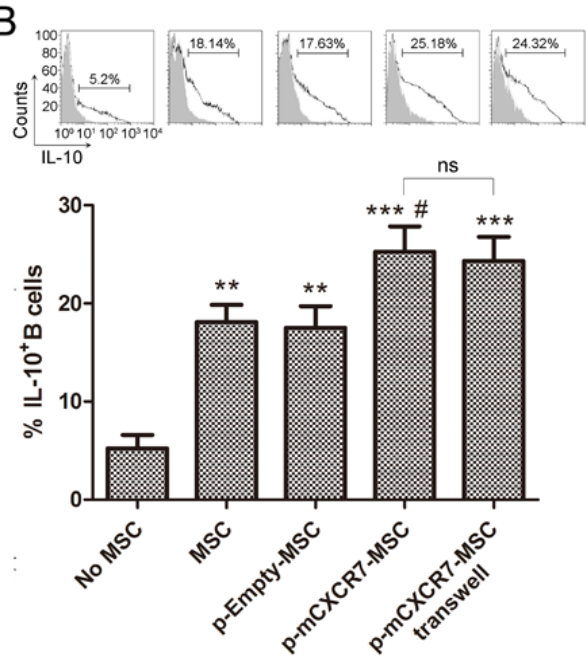

C

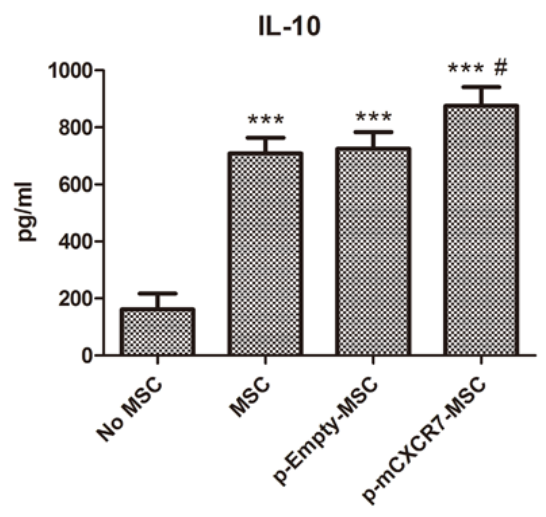

D

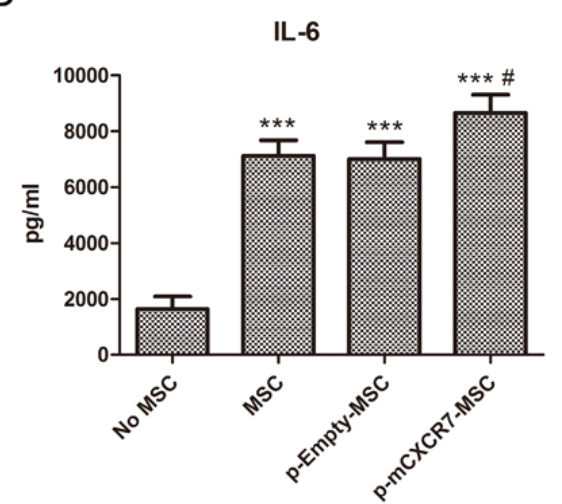

Fig. 6. Effects of CXCR7overexpression on MSC-induced IL-10+Breg cells. (A) MSCs were transiently transfected with using the pUNO1-mCXCR7 vector overexpressing CXCR7 (p-mCXCR7) or pUNO1-Empty vector (p-Empty). ELISA was performed to assess MSC secretion. * vs the control group. CD19+B cells were incubated at a 1:1 ratio with MSCs, CXCR7-overexpressing MSCs (p-mCXCR7-MSC) or p-Empty-MSC in a transwell experiment. B cells were stained with anti-CD19 in combination with intracellular staining for IL-10 and analyzed with flow cytometry. (B) Representative flow cytometric plots and percentages of IL-10 $0^{+}$B cells are shown. Culture supernatants were subsequently collected for IL-10 (C) and IL-6 (D) quantification using ELISA. Data are presented as mean \pm SD $(n=3)$.* vs the 'no MSC' group; \# vs the B cell : MSC group; ns, not statistically significant. ${ }^{*}, \# \mathrm{p}<0.05,{ }^{* *}, \mathrm{p}<0.01,{ }^{* * *}, \mathrm{p}<0.001$ (all panels).

nor AMD3100 effected the decrease in cell viability and increase in LDH release induced at high SDF- $1 \alpha$ concentrations $(1 \mu \mathrm{g} / \mathrm{ml})$ (Fig. $3 \mathrm{H}$ and I).

To ascertain whether decreasing CXCR7 levels at high SDF- $1 \alpha$ concentrations affect MSC proliferation and viability, CXCR7 was transiently overexpressed in MSCs. Transient overexpression of CXCR7 in MSCs prior to treatment with SDF-1 $\alpha(1 \mu \mathrm{g} / \mathrm{ml})$ promoted cell proliferation and induced an increase in cell viability and decrease in LDH release (Fig. 3J, $\mathrm{K}$ and $\mathrm{L}$ ). Our results clearly indicate that the CXCR7 receptor is required for MSC viability under SDF- $1 \alpha$ culture conditions.

Effect of SDF-1 $\alpha$-CXCR7 on the immunomodulatory activity of MSCs on IL-10+Breg cells

MSCs secrete factors that regulate immunity. Low concentrations of SDF- $1 \alpha(0.1 \mu \mathrm{g} / \mathrm{ml})$ induced a significantly increase in MSC-secreted IL- 6 , IL-10, IL-4, IFN- $\gamma$, and TNF- $\alpha$, compared with the control group. At high concentrations of SDF- $1 \alpha(\geq 0.5 \mu \mathrm{g} / \mathrm{ml})$, cytokine levels started to decrease gradually (Fig. 4A). Secretion of cytokines induced by low concentrations of SDF- 
$1 \alpha(0.1 \mu \mathrm{g} / \mathrm{ml})$ was completely abolished by the CXCR7 receptor inhibitor, CCX771. Moreover, CXCR7 overexpression in MSCs reversed the inhibitory effect of high concentrations of SDF$1 \alpha(1 \mu \mathrm{g} / \mathrm{ml})$ (Fig. 5A). Base on the results, we suggest that the CXCR7 receptor is required for MSC paracrine action under SDF- $1 \alpha$ culture conditions.

To establish whether the SDF-1-CXCR7 axis plays a role in MSC immunoregulation, MSCs were pretreated with the indicated concentrations of SDF- $1 \alpha$ for $24 \mathrm{~h}$ and co-cultured with B cells for 3 days. Low concentrations of SDF- $1 \alpha(0.1 \mu \mathrm{g} / \mathrm{ml}$ ) (cultured at a 1:1 ratio with MSCs) promoted the immunomodulatory effect of MSCs, inducing a further increase in IL-10+Breg cells (Fig. 4B, C) and IL-10 secretion production (Fig. 4D), relative to the levels observed in the absence of SDF- $1 \alpha$. However, at high concentrations, SDF- $1 \alpha(\geq 0.5 \mu \mathrm{g} / \mathrm{ml})$ inhibited the immunomodulatory effect of MSCs on B cells, and the increase in IL-10 $0^{+}$Breg cells as well as IL-10 and IL-6 secretion were significantly suppressed (Fig. 4B-E). Low SDF$1 \alpha$-induced secretion of cytokines $(0.1 \mu \mathrm{g} / \mathrm{ml})$ was completely abolished by CXCR7 receptor inhibitor, CCX771. Overexpression of CXCR7 in MSCs reversed the inhibitory effect of high concentrations of SDF- $1 \alpha$ (Fig. 5C-E).

Effects of CXCR7-overexpressing MSCS on B-cell subsets are dependent on soluble factors

Secretion of soluble factors (except IL-7) was significantly increased in CXCR7overexpressing MSCs, compared with control (Fig. 6A). To determine whether release of these soluble factors is necessary for B-cell stimulation, we stimulated B-cell subsets in a transwell system with B cells in the lower chamber and CXCR7-overexpressing MSCs in the upper chamber (1:1 ratio). In parallel, control B cells were kept in contact with MSCs, similar to the above experiments. A significant increase in IL-10+Breg cells was induced by CXCR7overexpressing MSCs irrespective of the presence or absence of the filter separating B cells from MSCs (Fig. 6B). CXCR7-overexpressing cells also promoted secretion of IL-10 and IL-6 into the co-culture supernatant (Fig. 6C and D).

\section{Discussion}

MSCs have been shown to exert immunosuppressive activity in vitro and in vivo on activated T cells [30], dendritic cells [33] and NK cells [34]. However, conflicting results have been obtained with regard to direct immunomodulatory effects of MSCs on B cells, with previous studies reporting two opposite effects on B cell proliferation and differentiation. Krampera et al. [11] showed that MSCs inhibited the B-cell proliferation, dependent on IFN$\gamma$-induced indoleamine 2,3-dioxygenase (IDO) production. Corcione and co-workers [10] demonstrated that in vitro, MSCs suppress proliferation of B cells activated with different B-cell tropic stimuli and interfere with their differentiation, chemotactic behavior and antibody production. In contrast, Traggiai et al. [9] reported that bone marrow-derived MSCs in vitro are able to promote proliferation and differentiation of transitional and naive B cells upon stimulation with CpG, soluble CD40L, anti-Ig antibodies and IL-2. These discrepancies may be attributable to various factors, including different experimental conditions, species of the MSC origin, purity of B cells or MSCs, strength of stimuli, and different signaling pathways initiated by the stimuli via the cell-cell contact or humoral factors. Further investigate is essential to verify the immunomodulatory action of MSCs on B cells.

In the current study we evaluated the immunomodulatory effects of MSCs on Breg cells. CD19+ cells co-cultured with MSCs at equal or 3 to 4 -fold higher counts resulted in stimulation of intracellular IL-10 expression in B-cells and increased secretion of IL-10, IL-6, and IgM. In contrast, we observed limited production of cytokines (IL-6 and IL-10) in cultures of MSC alone. Incresed production of IL-10 and IL- 6 in co-culture supernatants was mainly attributed to secretion by B-cells. In addition, we demonstrated that MSCs primarily induced CD1 $\mathrm{d}^{+}$MZ B cells to generate IL-10, consistent with previous studies showing that MZ B cells are the major IL-10-producing B cells [35]. CD1d expression may be associated with the increase in IL-10 production [36]. The Breg subset, characterized by increased 
CD1d expression, was initially identified by Mizoguchi et al. [37]. The group showed that CD1 $\mathrm{d}^{\text {hi }} \mathrm{B}$ cells producing IL-10 act as regulatory cells to suppress the progression of intestinal inflammation in a mouse model of chronic colitis. Future studies should focus on the precise working mechanism underlying the regulatory role of MSC-induced B cells.

The immunomodulatory mode of action of MSCs remains unclear at present. Previously, Rassmuson and colleagues [5] reported contact-dependent stimulation of IgG production in spleen-derived B-cell by MSCs. The group also reported MSC stimulation of IL-6 and INF- $\gamma$ in spleen-derived mononuclear cells, although they did not observe this effect on B-cell enriched cultures. A number of studies have shown that MSCs regulate T-cell immune functions by secreting a variety of cytokines and chemokines, such as IL-10, IL- 6 , IFN- $\gamma$, TNF- $\alpha$, TGF- $\beta$, PGE2, and HGF $[4,18,38]$. Data from the current study indicate that SDF- $1 \alpha$ regulates MSC viability and paracrine actions through CXCR7. In our experiments, low concentrations of SDF- $1 \alpha(0.1 \mu \mathrm{g} / \mathrm{ml})$ promoted MSC proliferation and production of cytokines (IL-6, IL-10, IL-4, IFN- $\gamma$, TNF- $\alpha$ ) through CXCR7, possibly stimulating the regulatory function of B cell. However, higher concentrations of SDF- $1 \alpha(\geq 0.5 \mu \mathrm{g} / \mathrm{ml})$ led to significantly decreased MSC cell viability and increased cytotoxicity. At high concentrations of SDF- $1 \alpha(\geq 0.5 \mu \mathrm{g} / \mathrm{ml})$, levels of MSC cytokines (IL-6, IL-10, IL-4, IFN- $\gamma$, TNF- $\alpha$ ) were markedly lower than those at low concentrations of SDF- $1 \alpha$. Notably, we observed a clear reduction of intracellular IL-10 expression and secretion of IL-10 and IL- 6 in CD19 ${ }^{+}$cells co-cultured with MSCs. Consistent with the reported CXCR7 scavenger activity [27, 39], our results showed that overexpression of CXCR7 reverses the effects of SDF-1. Data from transwell assays additionally showed that the effect of CXCR7-overexpressing MSCs on B-cell subsets is dependent on soluble factors. CXCR7 overexpression significantly enhanced the secretion of these factors in MSCs. Previous studies have shown that IL-6, a potent B-cell growth factor, is produced by MSCs after stimulation with TLR9 agonist. IFN- $\gamma$ promotes the immunosuppressive capacity of adult human mesenchymal stem cells [40]. CXCR7 might serve as a promising candidate for Breg stimulation by MSCs. The findings suggested that dual directional regulation between SDF-1a and CXCR7, although the exact molecular mechanism requires further investigation.

In conclusion, we have demonstrated that an immunomodulatory effect of MSCs on the activation of murine B lymphocytes stimulated with LPS and $\alpha$ CD40 and induction of Breg cells by MSCs for the first time. However, the immune actions of MSC-induced Bregs in vitro and in vivo require elucidation. In addition, the SDF-1-CXCR7 axis appears to play an important role in Breg stimulation by MSCs via paracrine actions, presenting a potential candidate target to enhance the immunomodulatory function of these cells.

\section{Acknowledgements}

This work was supported by the Shanghai Academic Leaders' Program of the Health System (No. XBR2011038).

\section{Disclosure Statement}

None.

\section{References}

1 Bruder SP, Fink DJ, Caplan AI: Mesenchymal stem cells in bone development, bone repair, and skeletal regeneration therapy. J Cell Biochem 1994;56:283-294.

2 Zhao S, Wehner R, Bornhauser M, Wassmuth R, Bachmann M, Schmitz M: Immunomodulatory properties of mesenchymal stromal cells and their therapeutic consequences for immune-mediated disorders. Stem Cells Dev 2009;19:607-614. 


\section{Cellular Physiology Cell Physiol Biochem 2015;37:117-130 \begin{tabular}{l|l|l} 
DOI: 10.1159/000430338 & (C) 2015 S. Karger AG, Basel
\end{tabular} \begin{tabular}{l|l} 
and Biochemistry Published online: August 17, 2015 & www.karger.com/cpb \\
\cline { 1 - 1 }
\end{tabular}

3 Franquesa M, Herrero E, Torras J, Ripoll E, Flaquer M, Goma M, Lloberas N, Anegon I, Cruzado JM, Grinyo JM, Herrero-Fresneda I: Mesenchymal stem cell therapy prevents interstitial fibrosis and tubular atrophy in a rat kidney allograft model. Stem Cells Dev 2012;21:3125-3135.

$4 \quad$ Nauta AJ, Fibbe WE: Immunomodulatory properties of mesenchymal stromal cells. Blood 2007;110:34993506.

5 Rasmusson I, Le Blanc K, Sundberg B, Ringden O: Mesenchymal stem cells stimulate antibody secretion in human b cells. Scand J Immunol 2007;65:336-343.

6 Rasmusson I, Uhlin M, Le Blanc K, Levitsky V: Mesenchymal stem cells fail to trigger effector functions of cytotoxic t lymphocytes. J Leukoc Biol 2007;82:887-893.

7 Bassi EJ, Aita CA, Camara NO: Immune regulatory properties of multipotent mesenchymal stromal cells: Where do we stand? World J Stem Cells 2011;3:1-8.

8 Franquesa M, Hoogduijn MJ, Bestard 0, Grinyo JM: Immunomodulatory effect of mesenchymal stem cells on b cells. Front Immunol 2012;3:212.

9 Traggiai E, Volpi S, Schena F, Gattorno M, Ferlito F, Moretta L, Martini A: Bone marrow-derived mesenchymal stem cells induce both polyclonal expansion and differentiation of $\mathrm{b}$ cells isolated from healthy donors and systemic lupus erythematosus patients. Stem Cells 2008;26:562-569.

10 Corcione A, Benvenuto F, Ferretti E, Giunti D, Cappiello V, Cazzanti F, Risso M, Gualandi F, Mancardi GL, Pistoia V, Uccelli A: Human mesenchymal stem cells modulate b-cell functions. Blood 2006;107:367-372.

11 Krampera M, Cosmi L, Angeli R, Pasini A, Liotta F, Andreini A, Santarlasci V, Mazzinghi B, Pizzolo G, Vinante F, Romagnani P, Maggi E, Romagnani S, Annunziato F: Role for interferon-gamma in the immunomodulatory activity of human bone marrow mesenchymal stem cells. Stem Cells 2006;24:386-398.

12 Mauri C, Bosma A: Immune regulatory function of b cells. Annu Rev Immunol 2012;30:221-241.

13 Evans JG, Chavez-Rueda KA, Eddaoudi A, Meyer-Bahlburg A, Rawlings DJ, Ehrenstein MR, Mauri C: Novel suppressive function of transitional $2 \mathrm{~b}$ cells in experimental arthritis. J Immunol 2007;178:7868-7878.

14 DiLillo DJ, Matsushita T, Tedder TF: B10 cells and regulatory b cells balance immune responses during inflammation, autoimmunity, and cancer. Ann N Y Acad Sci 2010;1183:38-57.

15 Yanaba K, Bouaziz JD, Haas KM, Poe JC, Fujimoto M, Tedder TF: A regulatory b cell subset with a unique cd1dhicd5+ phenotype controls t cell-dependent inflammatory responses. Immunity 2008;28:639-650.

16 Yanaba K, Bouaziz JD, Matsushita T, Tsubata T, Tedder TF: The development and function of regulatory b cells expressing il-10 (b10 cells) requires antigen receptor diversity and tlr signals. J Immunol 2009;182:7459-7472.

17 Mizoguchi A, Bhan AK: A case for regulatory b cells. J Immunol 2006;176:705-710.

18 Yagi H, Soto-Gutierrez A, Parekkadan B, Kitagawa Y, Tompkins RG, Kobayashi N, Yarmush ML: Mesenchymal stem cells: Mechanisms of immunomodulation and homing. Cell Transplant 2010;19:667-679.

19 Uccelli A, Pistoia V, Moretta L: Mesenchymal stem cells: A new strategy for immunosuppression? Trends Immunol 2007;28:219-226.

20 Miller RJ, Banisadr G, Bhattacharyya BJ: Cxcr4 signaling in the regulation of stem cell migration and development. J Neuroimmunol 2008;198:31-38.

21 Vandercappellen J, Van Damme J, Struyf S: The role of cxc chemokines and their receptors in cancer. Cancer Lett 2008;267:226-244.

22 Vinader V, Afarinkia K: The emerging role of cxc chemokines and their receptors in cancer. Future Med Chem 2012;4:853-867.

23 Brunn A, Utermohlen O, Mihelcic M, Sanchez-Ruiz M, Carstov M, Blau T, Ustinova I, Penfold M, MontesinosRongen M, Deckert M: Differential effects of cxcr4-cxcl12- and cxcr7-cxcl12-mediated immune reactions on murine p0106-125 -induced experimental autoimmune neuritis. Neuropathol Appl Neurobiol 2013;39:772-787.

24 Busillo JM, Benovic JL: Regulation of cxcr4 signaling. Biochim Biophys Acta 2007; 1768:952-963.

25 Lagane B, Chow KY, Balabanian K, Levoye A, Harriague J, Planchenault T, Baleux F, Gunera-Saad N, Arenzana-Seisdedos F, Bachelerie F: Cxcr4 dimerization and beta-arrestin-mediated signaling account for the enhanced chemotaxis to cxcl12 in whim syndrome. Blood 2008;112:34-44.

26 Xu FT, Li HM, Yin QS, Liu DL, Nan H, Zhao PR, Liang SW: Human breast adipose-derived stem cells transfected with the stromal cell-derived factor-1 receptor cxcr4 exhibit enhanced viability in human autologous free fat grafts. Cell Physiol Biochem 2014;34:2091-2104. 


\section{Cellular Physiology Cell Physiol Biochem 2015;37:117-130 \begin{tabular}{l|l} 
DOI: 10.1159/000430338 & (C) 2015 S. Karger AG, Basel
\end{tabular} \\ Qin et al.: MSCs Induce IL10+ Breg Cells}

27 Naumann U, Cameroni E, Pruenster M, Mahabaleshwar H, Raz E, Zerwes HG, Rot A, Thelen M: Cxcr7 functions as a scavenger for cxcl12 and cxcl11. PLoS One 2010;5:e9175.

28 Humpert ML, Pinto D, Jarrossay D, Thelen M: Cxcr7 influences the migration of b cells during maturation. Eur J Immunol 2013;44:694-705.

29 Luz-Crawford P, Kurte M, Bravo-Alegria J, Contreras R, Nova-Lamperti E, Tejedor G, Noel D, Jorgensen C, Figueroa F, Djouad F, Carrion F: Mesenchymal stem cells generate a cd4+cd25+foxp3+ regulatory t cell population during the differentiation process of th1 and th17 cells. Stem Cell Res Ther 2013;4:65.

30 Carrion F, Nova E, Luz P, Apablaza F, Figueroa F: Opposing effect of mesenchymal stem cells on th1 and th17 cell polarization according to the state of cd4+ t cell activation. Immunol Lett 2010;135:10-16.

31 Kuan YC, Wu YJ, Hung CL, Sheu F: Trametes versicolor protein yzp activates regulatory b lymphocytes gene identification through de novo assembly and function analysis in a murine acute colitis model. PLoS One 2013;8:e72422.

32 Calpe E, Purroy N, Carpio C, Abrisqueta P, Carabia J, Palacio C, Castellvi J, Crespo M, Bosch F: Zap-70 promotes the infiltration of malignant b-lymphocytes into the bone marrow by enhancing signaling and migration after cxcr4 stimulation. PLoS One 2013;8:e81221.

33 Spaggiari GM, Abdelrazik H, Becchetti F, Moretta L: Mscs inhibit monocyte-derived dc maturation and function by selectively interfering with the generation of immature dcs: Central role of msc-derived prostaglandin e2. Blood 2009;113:6576-6583.

34 Spaggiari GM, Capobianco A, Abdelrazik H, Becchetti F, Mingari MC, Moretta L: Mesenchymal stem cells inhibit natural killer-cell proliferation, cytotoxicity, and cytokine production: Role of indoleamine 2,3-dioxygenase and prostaglandin e2. Blood 2008;111:1327-1333.

35 Barr TA, Brown S, Ryan G, Zhao J, Gray D: Tlr-mediated stimulation of apc: Distinct cytokine responses of b cells and dendritic cells. Eur J Immunol 2007;37:3040-3053.

36 Colgan SP, Hershberg RM, Furuta GT, Blumberg RS: Ligation of intestinal epithelial cd1d induces bioactive il-10: Critical role of the cytoplasmic tail in autocrine signaling. Proc Natl Acad Sci U S A 1999;96:1393813943.

37 Mizoguchi A, Mizoguchi E, Takedatsu H, Blumberg RS, Bhan AK: Chronic intestinal inflammatory condition generates il-10-producing regulatory b cell subset characterized by cd1d upregulation. Immunity 2002;16:219-230.

38 Locatelli F, Maccario R, Frassoni F: Mesenchymal stromal cells, from indifferent spectators to principal actors. Are we going to witness a revolution in the scenario of allograft and immune-mediated disorders? Haematologica 2007;92:872-877.

39 Berahovich RD, Zabel BA, Lewen S, Walters MJ, Ebsworth K, Wang Y, Jaen JC, Schall TJ: Endothelial expression of cxcr7 and the regulation of systemic cxcl12 levels. Immunology 2013;141:111-122.

40 Ryan JM, Barry F, Murphy JM, Mahon BP: Interferon-gamma does not break, but promotes the immunosuppressive capacity of adult human mesenchymal stem cells. Clin Exp Immunol 2007;149:353363. 